\title{
Sudden hearing loss in a melanoma patient on pembrolizumab: an etiology not to be omitted in the differential diagnosis
}

\author{
Marc-Elie Nader ${ }^{*}$ D, Jeffrey N. Myers and Paul W. Gidley
}

\begin{abstract}
Immune checkpoint inhibitors have emerged as a promising therapeutic option for metastatic cancers. However, they have been associated with inflammatory adverse reactions in various organ systems. A recent article reported a case of sudden bilateral hearing loss that occurred in a patient with metastatic melanoma being treated with pembrolizumab. The authors attributed that complication to an autoimmune reaction secondary to the treatment. This commentary discusses the importance of considering the diagnosis of leptomeningeal metastasis in patients with metastatic melanoma who present with new cranial nerve deficits.
\end{abstract}

Keywords: Immune checkpoint inhibitor, Pembrolizumab, Melanoma, Hearing loss, Leptomeningeal metastasis

\section{Main text}

We read with great interest the paper by Zibelman and collaborators titled "Autoimmune inner ear disease in a melanoma patient treated with pembrolizumab" published in the Journal for ImmunoTherapy of Cancer [1]. The authors report a patient with widespread metastatic mucosal melanoma who developed sudden bilateral hearing loss while undergoing treatment with pembrolizumab. The episode of hearing loss was thought to be an autoimmune inner ear event secondary to the use of the immune checkpoint inhibitor.

Autoimmune inner ear disease (AIED) is defined as rapidly progressive, usually asymmetric sensorineural hearing loss [2]. Definitive tests do not exist to make this diagnosis, but a proportion of patients with AIED have antibodies against myelin P0, cochlin, $\beta$-tectorin and HSP-70 [2]. The diagnosis is often based on a careful history, physical examination, audiometry, laboratory tests and diagnostic imaging, excluding other etiologies of hearing loss.

Given that the patient had a melanoma of the anterior skull base, we respectfully point out that another etiology, namely leptomeningeal metastasis (LM), should have also been considered in the differential diagnosis for this episode

\footnotetext{
* Correspondence: mnader@mdanderson.org

Department of Head and Neck Surgery, Unit 1445, The University of Texas MD Anderson Cancer Center, 1515 Holcombe Blvd., Houston, TX 77030, USA
}

of hearing loss. After carefully reading the article, it was not readily apparent if the authors had ruled out that potential cause. LM can complicate any cancer and is seen in up to $8 \%$ of all cancer patients. Melanoma is among the three most common types of malignancies that cause LM [3]. Bilateral sudden onset hearing loss has been described in the context of LM in several reports, including a patient with melanoma [4-6].

A high level of suspicion for LM must be kept in mind for patients with metastatic carcinomas who present with new cranial nerve deficits or other neurological symptoms. The diagnosis of LM may be difficult to establish. Magnetic resonance imaging (MRI) of the brain with gadolinium is the imaging study of choice. LM can sometimes be microscopic and may not show on MRI. In that case, lumbar puncture (LP) with demonstration of malignant cells in the cerebrospinal fluid can confirm the diagnosis [3]. It is unclear if the patient reported in this article underwent an MRI of the brain or an LP once hearing loss was confirmed.

It has been recently suggested that LM from melanoma may respond to systemic targeted therapy and immune checkpoint inhibitors [7]. This finding raises the possibility that the hearing loss in this patient was, in fact, caused by LM and the improvement in hearing resulted from a positive response to pembrolizumab and a decrease in disease burden. However, as the authors pointed out, there exists 
a potential for checkpoint inhibitors to be associated with AIED as they have been with other autoimmune related neurologic complications. It is important to remain vigilant about this possibility and to refer patients for appropriate testing and management if that pathology is suspected.

\section{Authors' response}

Matthew Zibelman, Natasha Pollak and Anthony J. Olszanski

We would like to thank Nader et al for their response to our case report entitled "Autoimmune inner ear disease in a melanoma patient treated with pembrolizumab." We agree that development of leptomeningeal disease (LMD) is an important diagnostic dilemma for patients with metastatic melanoma and should be high on the differential in patients with this disease who develop new cranial nerve deficits, including hearing loss. As the authors point out, bilateral hearing loss has been described in association with LMD, although this would be quite rare, particularly without other detectable cranial nerve deficits. Bilateral hearing loss would be theoretically more likely as an unintended, on-target side effect of cross-reactive activated T-cells, though further reports of incidence as anti-programmed death 1 (PD-1) pathway drugs become more prevalent would be informative. In our patient, he did not have magnetic resonance imaging (MRI) of the brain initially, but that was a function of his claustrophobia. He refused MRI evaluation, thus we proceeded with alternative assessments. Given his presentation at that time with no other central nervous system (CNS) deficits, clinical improvement with PD-1 blockade, and evidence of bilateral sensorineural hearing loss, intratympanic dexamethasone injections were felt to be both diagnostic and potentially therapeutic. The patient did subsequently agree to have an MRI of his brain 6 months later and it did in fact confirm an absence of LMD or other CNS disease. The patient remains well, without evidence of progressive disease, now 14 months since discontinuation of the PD-1 inhibitor. In this patient, the strong temporal association between the development of bilateral hearing loss coincident with anti-PD-1 therapy, with significant resolution of hearing impairment upon initiation of intratympanic steroid administration, strongly suggests that the hearing loss is associated with anti-PD-1 therapy.

\section{Abbreviations}

AIED: Autoimmune inner ear disease; LM: Leptomeningeal metastasis: LP: Lumbar puncture; MRI: Magnetic resonance imaging

\section{Acknowledgements}

Not applicable.

Funding

Not applicable.

Availability of data and materials Not applicable.
Authors' contributions

All authors drafted, read and approved this letter. MEN, JNM, PWG.

Competing interests

The authors declare that they have no competing interests.

Consent for publication

We hereby consent to the publication of this manuscript.

Ethics approval and consent to participate

Not applicable.

Received: 9 January 2017 Accepted: 15 February 2017

Published online: 21 March 2017

References

1. Zibelman M, Pollak N, Olszanski AJ. Autoimmune inner ear disease in a melanoma patient treated with pembrolizumab. J Immunother Cancer. 2016;4:8.

2. Goodall AF, Siddiq MA. Current understanding of the pathogenesis of autoimmune inner ear disease: a review. Clin Otolaryngol. 2015;40(5):412-9.

3. DeAngelis LM, Boutros D. Leptomeningeal metastasis. Cancer Invest. 2005; 23(2):145-54.

4. Jeffs GJ, Lee GY, Wong GT. Leptomeningeal carcinomatosis: an unusual cause of sudden onset bilateral sensorineural hearing loss. J Clin Neurosci. 2006;13(1):116-8.

5. Uppal HS, Ayshford CA, Wilson F. Sudden onset bilateral sensorineural hearing loss: a manifestation of occult breast carcinoma. J Laryngol Otol. 2001;115:907-11.

6. Civantos F, Choi YS, Applebaum EL. Meningeal carcinomatosis producing sudden hearing loss: a case report. Am J Otol. 1992;13:369-71.

7. Geukes Foppen MH, Brandsma D, Blank CU, van Thienen JV, Haanen JB, Boogerd W. Targeted treatment and immunotherapy in leptomeningeal metastases from melanoma. Ann Oncol. 2016;27(6):1138-42.

Submit your next manuscript to BioMed Central and we will help you at every step:

- We accept pre-submission inquiries

- Our selector tool helps you to find the most relevant journal

- We provide round the clock customer support

- Convenient online submission

- Thorough peer review

- Inclusion in PubMed and all major indexing services

- Maximum visibility for your research

Submit your manuscript at www.biomedcentral.com/submit 